\title{
Interstitial Lung Disease as an Adverse Drug Reaction in Japan: Exploration of Regulatory Actions as a Basis for High Reporting
}

\author{
Eiko Iwasa ${ }^{1} \cdot$ Yu Fujiyoshi ${ }^{1,3} \cdot$ Yuki Kubota $^{1} \cdot$ Ryota Kimura $^{1} \cdot$ Rebecca E. Chandler $^{2} \cdot$ Henric Taavola $^{2}$ (1) \\ G. Niklas Norén ${ }^{2} \cdot$ Rika Wakao' $^{1}$
}

Published online: 2 July 2020

(c) The Author(s) 2020

\begin{abstract}
Introduction Increased post-marketing reports of interstitial lung disease in Japan have been recognized. An understanding of its regional groundings can be important for the global pharmacovigilance community.

Objective The objective of this study was to explore the correlation between high rates of interstitial lung disease reporting and regulatory actions in Japan.

Methods Post-marketing interstitial lung disease-related label changes and interstitial lung disease reports were classified by the anatomical therapeutic chemical classification groups of the suspected drugs. Regulatory actions for the top interstitial lung disease-reporting drugs were compared. The interstitial lung disease reporting patterns of protein kinase inhibitors were compared to those of methotrexate.

Results Interstitial lung disease-related label changes predominantly occurred for drugs in the anatomical therapeutic chemical classification groups L, J, C, and herbal medicines. Interstitial lung disease was reported most frequently for L group, especially for the protein kinase inhibitors. The regulatory actions for those drugs with the highest number of interstitial lung disease reports (methotrexate, protease kinase inhibitors, gemcitabine, docetaxel) plus monoclonal antibodies were analyzed. The ratio of interstitial lung disease reports to all reports over time was initially high in the re-examination period, while it was constantly low after the period expired. The increase in interstitial lung disease reporting was observed for the drugs for which interstitial lung disease was designated as a priority item in the use-results survey. Methotrexate had more interstitial lung disease reports with multiple suspected drugs and fewer reports with high completeness than the protease kinase inhibitors.

Conclusions The high rates of interstitial lung disease reporting derived from mainly the anatomical therapeutic chemical classification group L drugs. Interstitial lung disease is the targeted adverse drug reaction in the use-results survey mandated in the re-examination of those drugs. This system provides at least one explanation for the high reporting of interstitial lung disease in Japan.
\end{abstract}

Disclaimer The views expressed in this article are the personal views of the authors and may not be understood or quoted as being made on behalf of or reflecting the position of the Pharmaceuticals and Medical Devices Agency and the World Health Organization.

Electronic supplementary material The online version of this article (https://doi.org/10.1007/s40264-020-00968-7) contains supplementary material, which is available to authorized users.

\section{Rika Wakao}

wakao-rika@pmda.go.jp

1 Pharmaceuticals and Medical Devices Agency (PMDA), 3-3-2 Kasumigaseki, Chiyoda-ku, Tokyo 100-0013, Japan

2 Uppsala Monitoring Centre, Uppsala, Sweden

3 Present Address: Kurume, Japan

\section{Key Points}

Increased post-marketing reports of interstitial lung disease in Japan have been recognized.

The high rates of interstitial lung disease reporting derived from mainly the anatomical therapeutic chemical classification group L drugs.

The regulatory system for those drugs may explain the high rates of interstitial lung disease reporting.

In particular, the impact of solicited reports from useresults survey is found. 


\section{Introduction}

In 1965, the World Health Organization recommended that member countries establish domestic drug monitoring systems. Accordingly, spontaneous adverse drug reaction (ADR) reporting started in Japan at designated hospitals in 1967. The reporting scope expanded to all medical institutions in 1997, and healthcare professional reporting was stipulated in the Pharmaceutical Affairs Law in 2003. Likewise, industry reporting started via administrative direction for new drugs in 1967 and expanded to all drugs in 1971. The Pharmaceutical Affairs Law was amended in 1979 because of the drug-induced SMON (Subacute MyeloOptico-Neuropathy) disease, stipulating mandatory reporting for the marketing authorization holders (MAHs) [1].

The re-examination system, established by the amended Pharmaceutical Affairs Law, is a unique system aimed at reconfirmation of the clinical usefulness of drugs at the end of the predetermined period after approval ("re-examination period"). When the predetermined period has passed (typically 8 years), re-examinations are conducted to confirm the effectiveness and safety, based on data on use results obtained by MAHs as shown in Fig. 1 [2]. The data sources used in the re-examination include spontaneous reports, post-marketing clinical trials, post-marketing database investigations, foreign regulatory action reports, and research reports. All case surveillance data, as a use-results survey, may also be a data source for those drugs whose approval has been based upon small-sized clinical trials or for which further data informing proper use are necessary. In addition, early phase post-marketing vigilance is conducted for most of the new drugs to promote proper use of the drug, detect serious adverse reactions earlier, and take safety measures immediately during the initial 6 months after the launch. The re-examination results categorize the drug into (1) regarded to be useful without any change in approval, (2) regarded to be useful with a partial change in approval, and (3) regarded not to be useful.

Interstitial lung disease (ILD) has been identified as an adverse event that has a higher reporting rate in Japan. In a previous analysis using Vigibase data, ILD was found to be one of the most outstanding features of Japanese ADR reporting compared with other countries [3]. Over-representation of Japanese reporting of ILD has previously been described by others $[4,5]$. International comparison of druginduced lung disease revealed markedly higher incidence rates in Japan than abroad in clinical trials and observational studies [6-8]. In a systematic review of drug-induced ILD, it was noted that more than one-third of the studies were from Japan, mainly large post-marketing studies [9].

It is possible that differences in pharmacogenomics, regional medical care, and social environment influence the risk, the detection, and the reporting of ADRs. However, it is also true that differences in regulatory practice may also be a potential contributor to high rates of ILD reporting. The aim of this investigation was to explore the relationship between high rates of ILD reporting and regulatory processes required for licensure in Japan.

\section{Materials and Methods}

\subsection{Interstitial Lung Disease-Related Drug Label Changes}

Interstitial lung disease-related changes in the "clinically significant adverse reactions" sections of the labeling for



Fig. 1 Diagram for the processes in the re-examination 
prescription drugs were examined between April 2002 and March 2017. Both the addition of ILD as a safety issue and changes to the risk description of ILD were counted; however, revisions for a given drug label were only counted once.

The drug classes were categorized using anatomical therapeutic chemical (ATC) classification codes according to the approved indications in Japan. Herbal medicines were separately classified for descriptive purposes in this analysis. The detailed procedure of the safety-related drug label changes is described in Ishiguro et al. [10].

\subsection{Adverse Drug Reaction Reports Collection}

Data recorded from April 2004 to February 2017 in the Japanese Adverse Drug Event Report (JADER) database were downloaded from the Pharmaceuticals and Medical Devices Agency (PMDA) website. In the JADER database, the cases are recorded in the Japanese fiscal year, which starts in April and ends in the following March. The time courses in all figures derived from the JADER database are presented in the Japanese fiscal year. During the re-examination period, both spontaneous reports and solicited reports are collected in parallel. The JADER database lists spontaneous reports from the MAHs and the healthcare professionals as well as solicited reports from studies such as the use-results survey. Nearly all cases in the JADER database are serious, as individual case safety reports on serious cases are subject to reporting to PMDA. Adverse events were extracted from the database using MedDRA ${ }^{\circledR}$ Preferred Terms 'Interstitial lung disease' and Standardized MedDRA Queries (SMQ) narrow 'Interstitial lung disease'. The suspected drugs of the extracted reports were classified according to the ATC classification system with the World Health Organization Drug Cross Reference Tool Japan [11].

\subsection{Regulatory Information}

The information for labels for the drugs as of July 2019 were collected from the PMDA website.

\subsection{Comparison Between Methotrexate and Protein Kinase Inhibitors}

To explore differences in reporting of ILD between drugs subject to different degrees of regulatory requirements, we performed an exploratory analysis using vigiPoint $[3,12]$. vigiPoint uses shrinkage odds ratios to identify substantial differences in relative reporting rates of different covariate values between two data subsets. The data set used for this analysis consisted of all Japanese reports between 2013 and 2017 (in reported years to VigiBase) inclusive in VigiBase, the World Health Organization global database of individual case safety reports. In these analyses, the dates of reports reflect when they were transferred to VigiBase from the member countries, grouped by calendar year.

\section{Results}

\subsection{Overview of the Regulatory Actions}

Interstitial lung disease-related label changes in the "clinically significant adverse reactions" sections of product labeling were made to 93 drugs from April 2002 to March 2017 (Fig. 2); 87 (92\%) of the changes involved the inclusion of ILD in the label; in the remaining $8 \%$, the changes involved further characterization of the previously described ILD risk. In ATC system level 1, the top four drug classes to undergo a label change were drugs for antineoplastic and immunomodulating agents (L; 22.6\%), anti-infectives for systemic use (J; 18.3\%), herbal medicines (18.3\%), and cardiovascular system $(\mathrm{C} ; 18.3 \%)$. Even though the most common drug class subject to label change was class $\mathrm{L}$ antineoplastic and immunomodulating agents, the labels for many drugs within this class contained information on the ILD risk at the time of the market launch.

Interstitial lung disease reports from April 2004 to February 2017 are presented according to the drug classes analyzed for the label change (Table 1). The number of ILD reports for herbal medicines were much less than L, C, A, and $\mathrm{J}$ drugs, although the label revisions for herbal medicines were frequent.

Other drug classes, such as A, C, and J, had a higher number of reports than herbal medicines. The typical examples of drug within classes were lansoprazole (214 cases) in class $\mathrm{A}$, amiodarone (602 cases) in class $\mathrm{C}$, and ribavirin (314 cases) in class $\mathrm{J}$, and the top reporting drug in herbal medicines was Saireito (103 cases). Accordingly, the ILD reports of each herbal medicine were much less compared with the ILD reports of other drugs.

To further clarify the distribution of ILD reports within the $\mathrm{L}$ drug class, the most frequently reported four suspected drug groups in ATC-code fourth level are shown in Fig. 3, PKIs (L01XE; 26\% of L01 group), pyrimidine analogs (L01BC; 16\%), monoclonal antibodies (L01XC; $13 \%$ ), and folic acid analogs (L01BA; 12\%). Of all drugs for which there were reports of ILD in the JADER database, methotrexate, a folic acid analog, had the highest total number of ILD reports and accounted for 84\% (1717 cases) of all reports in the L01BA group.

Table 2 lists the detailed information about labels for the top six drugs with the highest reporting of ILD, plus nivolumab. Nivolumab was identified as a key feature with higher rates of ILD reporting from Japan than from the rest of the world in a previous study [3]. The labels for these 


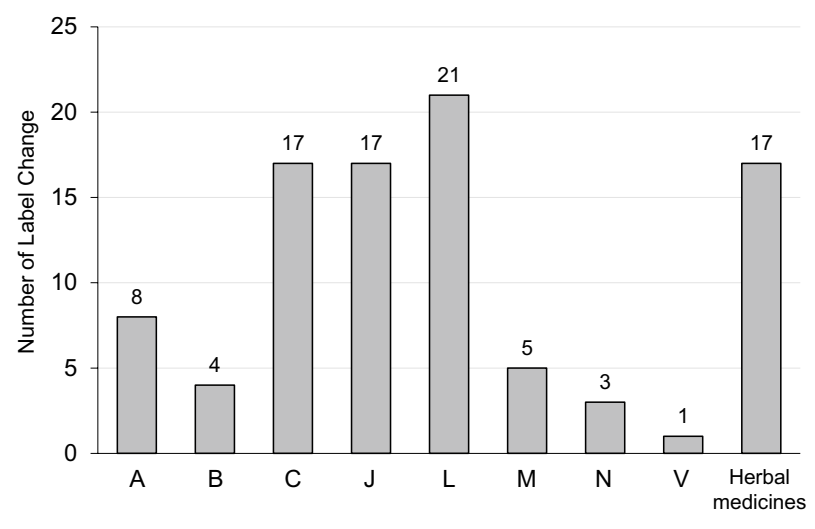

Fig. 2 Post-marketing safety label change for interstitial lung disease classified by suspected drugs [anatomical therapeutic chemical (ATC) classification system level 1]

Table 1 Interstitial lung disease reports in the drug classes shown in Fig. 2

\begin{tabular}{lrrr}
\hline $\begin{array}{l}\text { Suspected drugs (ATC } \\
\text { classification system } \\
\text { level 1) }\end{array}$ & Total & Fatal outcome & Non-fatal outcome \\
\hline A & 2604 & 472 & \\
B & 1083 & 195 & 8832 \\
C & 2858 & 530 & 2328 \\
J & 2502 & 416 & 2086 \\
L & 20,406 & 4323 & 16,083 \\
M & 1412 & 252 & 1160 \\
N & 1122 & 183 & 939 \\
V & 514 & 157 & 357 \\
Herbal medicines & 1177 & 88 & 1089 \\
\hline
\end{tabular}

ATC anatomical therapeutic chemical

drugs listed ILD risk information in the "clinically significant adverse reactions" section. In addition, ILD risk information was especially emphasized in the "warning", "careful administration", and "important precautions" sections of product labels.

\subsection{Reporting Patterns of Interstitial Lung Disease}

Reporting rates of ILD over time were analyzed in parallel with the regulatory requirements (Fig. 4a-g, Table 1 of the Electronic Supplementary Material). Representative drugs were selected from each of the highly reported ATC classes: methotrexate (folic acid analogs), gefitinib, everolimus, and erlotinib (PKIs), gemcitabine (pyrimidine analogs), and nivolumab (monoclonal antibodies).

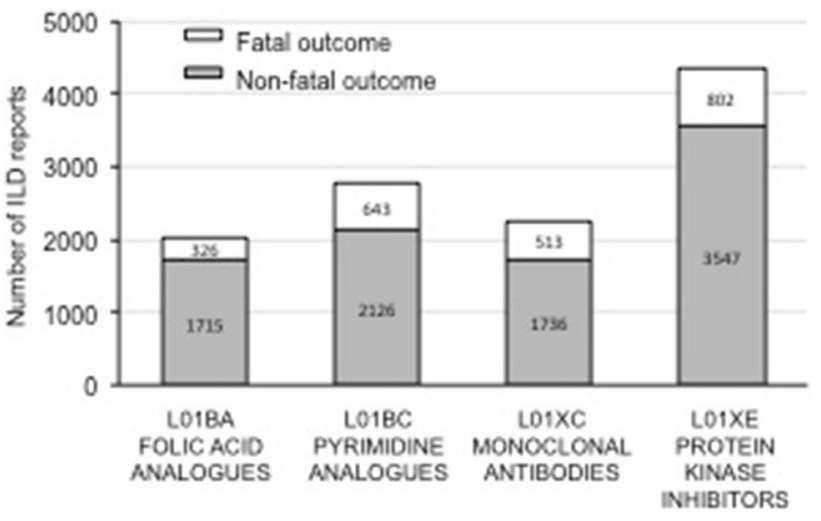

Fig. 3 Most frequently reported, four suspected drug groups in anatomical therapeutic chemical (ATC) classification system level 4 of interstitial lung disease (ILD) reports

Three types of reporting patterns were observed; constant (methotrexate, docetaxel, and gemcitabine), initial high followed by progressive decrease (gefitinib and erlotinib), and increase (everolimus and nivolumab). For methotrexate and docetaxel, ILD absolute reporting rates were constant throughout the observation period, 2004-2016 (Fig. 4a, d); important to note is that their re-examination periods had expired before 2004 (Table 1 of the ESM). For gemcitabine, ILD reporting was overall constant with a small increase limited in time near the end of the re-examination period from March 1999 to April 2011 (Fig. 4c). Gefitinib and erlotinib showed a pattern of initial high followed by progressive decrease (Fig. 4b, f). Placing these rates in relation to the re-examination periods for these drugs, it is suggested that reporting rates may initially increase at the beginning and progressively decline at the end.

Being in the early phase of the re-examination period, ILD reporting rate seems to be increasing for both everolimus and nivolumab (Fig. 4e, g). Although the data in the observation period were limited, the ILD reports for everolimus and nivolumab were similar in 2017 as in 2016 (data not shown). The ends of the re-examination periods for everolimus and nivolumab are not included in the observation period; it remains to be determined if rates will taper over time as these periods conclude in a similar pattern to gefitinib and erlotinib.

Interstitial lung disease is a group of more than 100 diseases but a previous study found the single Preferred Term ILD accounted for $85 \%$ of narrow SMQ ILD reports in Japanese reports [3]. A sensitivity analysis for the study at hand, based on the SMQ, showed closely correlated reporting rates over time of the Preferred Term for ILD with the narrow SMQ for ILD (Fig. 1 of the ESM).

As a complement to Fig. 4, we computed the ratio of ILD reports to all reports for each studied drug over time 
Table 2 Regulatory information for the top six drugs with highest reporting of interstitial lung disease (ILD), plus nivolumab

\begin{tabular}{|c|c|c|c|c|c|c|c|}
\hline & Methotrexate & Gefitinib & Gemcitabine & Docetaxel & Everolimus & Erlotinib & Nivolumab \\
\hline $\begin{array}{l}\text { Number of ILD total } \\
\text { case }\end{array}$ & 1717 & 1157 & 1064 & 886 & 833 & 808 & 436 \\
\hline Number of fatal case & 241 & 362 & 187 & 231 & 29 & 230 & 71 \\
\hline $\begin{array}{l}\text { Percentage of fatality } \\
(\%)\end{array}$ & 14 & 31 & 18 & 26 & 3.5 & 28 & 16 \\
\hline $\begin{array}{l}\text { Indication for lung } \\
\text { cancer }\end{array}$ & - & + & + & + & - & + & + \\
\hline \multicolumn{8}{|l|}{ Label description for ILD } \\
\hline & $\begin{array}{l}\text { For rheumatoid } \\
\text { arthritis (RA) }+\end{array}$ & & & & For cancer + & & \\
\hline \multirow[t]{2}{*}{ Warning } & & + & + & - & & + & + \\
\hline & $\begin{array}{l}\text { For cancer - } \\
\text { For RA + }\end{array}$ & & & & $\begin{array}{l}\text { For transplantation - } \\
\text { For cancer }+\end{array}$ & & \\
\hline \multirow[t]{2}{*}{$\begin{array}{l}\text { Careful administra- } \\
\text { tion }\end{array}$} & & + & + & + & & + & + \\
\hline & $\begin{array}{l}\text { For cancer - } \\
\text { For RA + }\end{array}$ & & & & $\begin{array}{l}\text { For transplantation - } \\
\text { For cancer }+\end{array}$ & & \\
\hline Important precautions & & + & + & - & & + & + \\
\hline $\begin{array}{l}\text { Clinically significant } \\
\text { adverse reactions }\end{array}$ & $\begin{array}{l}\text { For cancer - } \\
+\end{array}$ & + & + & + & $\begin{array}{l}\text { For transplantation - } \\
+\end{array}$ & + & + \\
\hline $\begin{array}{l}\text { Frequency described } \\
\text { in the label }\end{array}$ & $\begin{array}{l}0.1 \% \text { to less than } 5 \% \\
\text { for } \mathrm{RA}^{\mathrm{a}}\end{array}$ & $1 \%$ to less than $10 \%$ & $1.0 \%$ & $0.60 \%$ & $\begin{array}{l}15.1 \% \text { for cancer } \\
0.6 \% \text { for transplanta- } \\
\text { tion }\end{array}$ & $4.4 \%$ & Not described \\
\hline $\begin{array}{l}\text { Use-results survey } \\
\text { (which set ILD } \\
\text { as safety priority } \\
\text { items) }\end{array}$ & - & + & - & - & + & + & + \\
\hline
\end{tabular}

${ }^{a}$ Indicates methotrexate capsules

as shown in Fig. 5. Please note that some data points must be interpreted with caution as they are based on very low total numbers of reports for the drug (e.g., everolimus before 2010 and nivolumab in 2014). The ratios for gefitinib, methotrexate, and erlotinib show gradual decreases over time whereas the ratios for gemcitabine and docetaxel hover at more constant levels, and there is a suggestion of an increase for everolimus in 2015 and 2016. Even after the end of the re-examination periods, relative reporting rates of ILD remain higher for the studied drugs in Japan than in the rest of the world: the relative reporting rates of ILD for the studied drugs in VigiBase ${ }^{1}$ ranged between $0.5 \%$ for docetaxel and $4.1 \%$ for gefitinib.

\footnotetext{
${ }^{1}$ Data including all serious reports in VigiBase on 3 May, 2020 except suspected duplicates and reports from Japan; erlotinib $0.8 \%$, nivolumab $0.9 \%$, gemcitabine $1.0 \%$, everolimus $1.8 \%$, methotrexate $1.8 \%$.
}

\subsection{Comparison Between Interstitial Lung Disease Reports for Methotrexate and Protein Kinase Inhibitors}

The results of a vigiPoint comparison between ILD reports from Japan for methotrexate and protein kinase inhibitors are shown in Tables 3 and 4. Methotrexate had more reports with multiple suspected drugs and fewer reports with high completeness than PKIs. More ILD reports on PKIs were coming from studies than for methotrexate. There were more reports from physicians and fewer from patients for PKIs. For PKIs, there were about as many reports for men as for women, whereas for methotrexate there were about twice as many reports for women. No substantial differences in patient age were observed. 


\section{(a) Methotrexate}

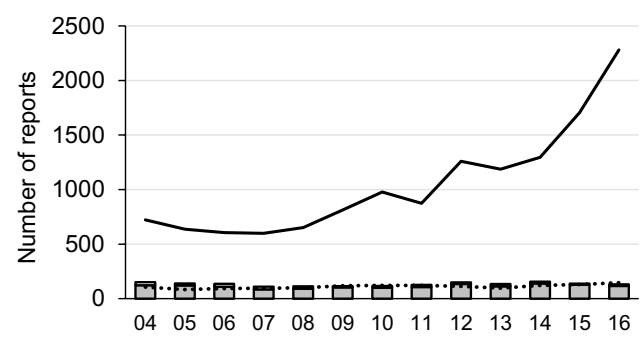

(c) Gemcitabine



(e) Everolimus

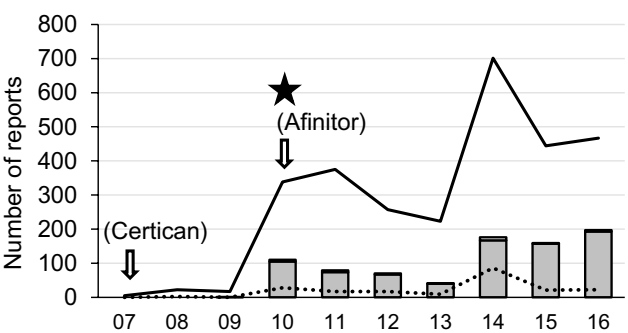

(g) Nivolumab

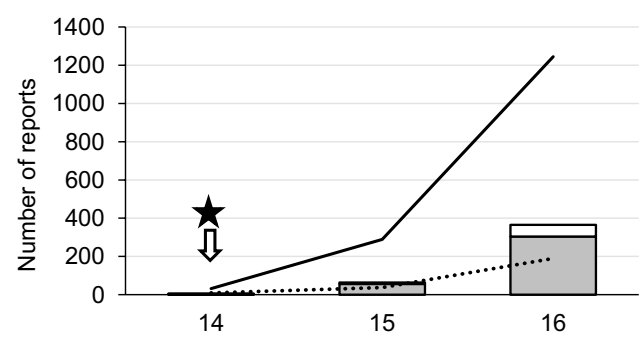

(b) Gefitinib

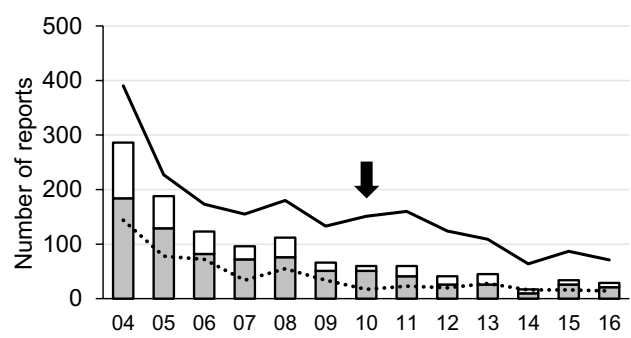

(d) Docetaxel

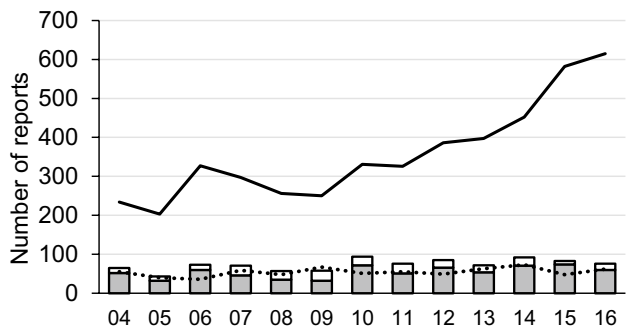

(f) Erlotinib

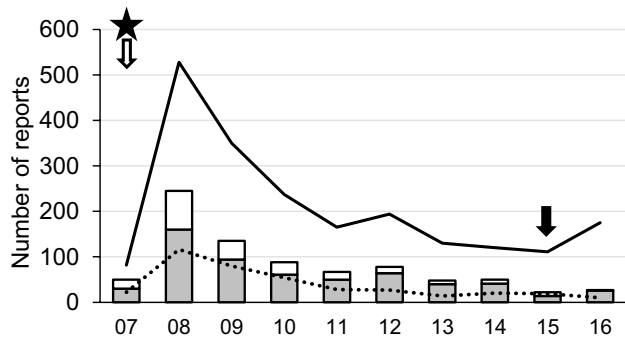

ILD fatal

ILD non-fatal

- all reports non-fatal

....... all reports fatal

$\pi$ the time point when the first re-examination period started

the time point when the latest re-examination period expired

the time point when the first use-result survey started which set ILD as safety priority item

Fig. 4 Frequency of interstitial lung disease (ILD) reporting of methotrexate (a), gefitinib (b), gemcitabine (c), docetaxel (d), everolimus (e), erlotinib (f), and nivolumab (g). The horizontal axis shows fiscal years as explained in the methods section 


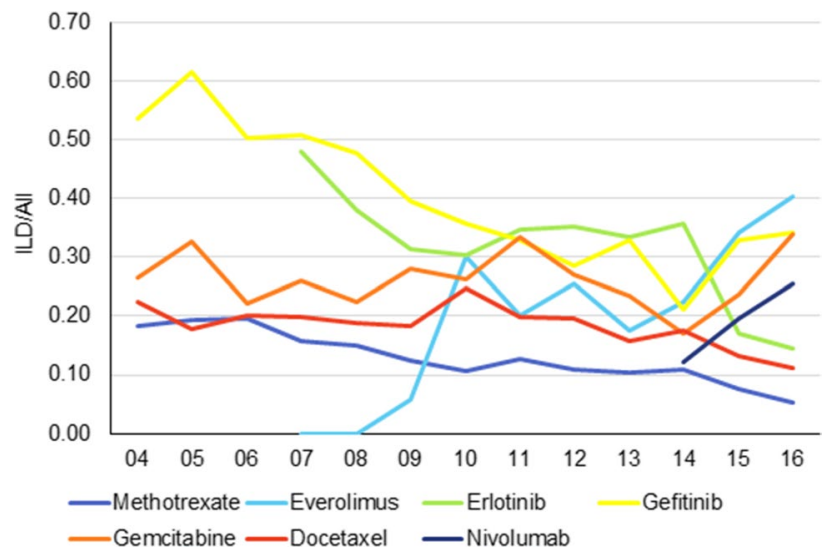

Fig. 5 Ratio of interstitial lung disease (ILD) reports to all reports over time for the seven drugs in Table 2

\section{Discussion}

In this study, we identified the high reporting of ILD derived from mainly the ATC group L (antineoplastic and immunomodulatory) drugs. Furthermore, there is evidence of increased reporting during the re-examination period. As ILD is a targeted ADR for these types of drugs in a regulatory mandated use-results survey, we conclude that pro-active regulatory requirements contribute to the high reporting of ILD observed in Japan.

\subsection{Grounds for High Rates of Reporting Interstitial Lung Disease}

\subsubsection{Role of the Use-Results Survey and Other Regulatory Aspects}

The use-results survey is specified to characterize the incidence and the risk factors for the target ADRs. A priority item is selected among the ADRs detected in the preapproval clinical trials based on a higher incidence and severity, and considerable attention is paid to the priority item in the survey during the re-examination. Interstitial
Table 4 Key features identified in the vigiPoint analysis, which have higher relative reporting rates on Japanese interstitial lung disease reports for protein kinase inhibitors (PKIs) than for methotrexate

\begin{tabular}{llll}
\hline Feature & $\begin{array}{l}\text { Methotrexate } \\
(n=691)(\%)\end{array}$ & $\begin{array}{l}\text { PKIs } \\
(n=2371) \\
(\%)\end{array}$ & Odds ratio \\
\hline $\begin{array}{l}\text { No. of suspected drugs per } \\
\text { report: } 1\end{array}$ & 54 & 89 & 0.15 \\
$\begin{array}{l}\text { Report type: from study } \\
\text { Reported by: physician }\end{array}$ & 11 & 32 & 0.26 \\
Completeness: high & 83 & 93 & 0.38 \\
Patient sex: male & 45 & 67 & 0.41 \\
\hline
\end{tabular}

lung disease was the priority item in the use-results survey that was mandated in the re-examination for gefitinib, erlotinib, everolimus, and nivolumab. These drugs revealed an increasing number of ILD reports after approval and/or high ratios of ILD reports to the overall reports. The ILD reports from the study for PKIs were $32 \%$ of the total ILD reports. In contrast, there were considerably fewer ILD reports of each herbal medicine compared with the top reporting L drugs. The herbal medicines are not subject to re-examination, because they are traditionally used with extensive clinical experience. Indeed, the labels for most of the herbal medicines have ILD mentioned only in the "clinically significant adverse reactions" section. The term ILD was added to their labels after use in a substantially larger and broader range of patient populations in the post-marketing period. Although there is a possibility of under-reporting in the spontaneous reports, the overall risk of ILD in the clinical setting of herbal medicine can be assumed to be much lower than in the oncology field. The patients with non-small cell lung cancer (NSCLC) who take antineoplastic drugs have often poor performance states and pulmonary fibrosis, which are the risk factors for life-threatening ILD, whereas the patients who take herbal medicines might be less susceptible, judging from their indications.

The solicited reporting process improves the quality of reports as compared with the spontaneous system, as standardized case report cards are utilized in the survey. While
Table 3 Key features identified in the vigiPoint analysis, which have higher relative reporting rates on Japanese interstitial lung disease reports for methotrexate than for protein kinase inhibitors (PKIs)

\begin{tabular}{llll}
\hline Feature & $\begin{array}{l}\text { Methotrexate } \\
(n=691)(\%)\end{array}$ & $\begin{array}{l}\text { PKIs }(n=2371) \\
(\%)\end{array}$ & Odds ratio \\
\hline Reported by: consumer/non-health professional & 6.0 & 1.1 & 5.9 \\
No. of suspected drugs per report: 2 & 36 & 8.9 & 5.7 \\
No. of suspected drugs per report: $3-5$ & 9.8 & 2.1 & 5.1 \\
Report type: spontaneous & 85 & 68 & 2.7 \\
Completeness: not high & 55 & 33 & 2.4 \\
Patient sex: female & 66 & 49 & 2.0 \\
Fatal: no & 87 & 79 & 1.7 \\
\hline
\end{tabular}


methotrexate has been the most frequently reported suspected drug of all drugs reporting ILD, their reports often contain scarce information in sharp contrast to PKIs. It is corroborated by the finding that the vigiPoint analysis revealed more ILD reports originating from studies on PKIs than on methotrexate. In addition, the early phase post-marketing vigilance that stimulates ADR reporting is required for new molecular entities. These regulatory processes for new drugs allow the capture of good-quality reports, which helps to make further regulatory decisions. The collected information of the new drug leads to the identification of risk factors and updates of product labels, which are shared with the labels for the generic drugs after the re-examination period expires.

Other regulatory processes may also stimulate ILD reporting. Inclusion in product labeling at the time of approval is suggested as one potential factor, as exemplified by the molecular-targeted drugs for NSCLC, specifically epidermal growth factor receptor (EGFR) inhibitors and tyrosine kinase inhibitors (TKIs). Many of them are found to induce ILD during their pre-approval clinical trials, resulting in the ILD risk being included in the label at the time of the market launch. The NSCLC drugs with the ILD warning tend to have high-reporting of ILD. In contrast, post-marketing labeling changes were found to affect reporting to a lesser extent as revealed by the example of herbal medicines in which there were comparable numbers of label changes in the observation period, but the accumulation of ILD reports was not high.

\subsubsection{High Attention to the Molecular-Targeted Drug}

Molecular-targeted drugs for NSCLC draw attention for a higher risk of ILD owing to a class effect of EGFR inhibitors and TKIs, as well as a higher incidence of pre-existing ILD in the disease [13]. Of note, the MAHs for EGFR-TKIs set independent ILD review committees of pulmonologists, chest radiologists, and pathologists to evaluate ILD-like cases to confirm ILD in the use-results survey $[14,15]$. It helped to standardize the detection of ILD and discovery of risk factors. A thorough examination of ILD-like cases could lead to detecting the precise occurrence.

Clinical practice can change during the re-examination period and this can be reflected in the reporting rates for ILD. Protein kinase inhibitors are subject to active surveillance in the re-examination period, as they are new molecular entities. During the average 8 years of re-examination, clinical practice can shift, such as was observed with EGFR-TKI. The emergency safety information (Yellow Letter) issued in 2002 to warn about life-threatening ILD changed the clinical use of gefitinib; after the Yellow Letter, it was more carefully administered, and more suitable patients selected. The warning sections of labels for gefitinib and erlotinib indicate "close monitoring of the onset of serious adverse reactions such as interstitial pneumonia etc. under hospitalization or under similar conditions for at least 4 weeks after the start of administration", despite the widely held view that solid oral dosage forms are used in ambulatory settings. In addition, the risk factors for EGFR-TKIs, such as a history of smoking, pre-existing pulmonary fibrosis, or poor functional status, were gradually elucidated [13], and chest specialists in general became well informed [16]. The use of EGFRTKIs has been avoided for patients with risk factors. Those precautions could contribute to the decrease in ILD reporting in the latter half of the re-examination period.

The amount of gefitinib used in 2004 could be high judging from the high reporting of non-ILD ADRs, while the reporting of non-ILD ADRs were constant during 2005 and 2013, suggesting that the used amounts did not decrease. The ratio of ILD to all reports for gefitinib decreased in those periods. Likewise, reporting of non-ILD ADRs for erlotinib decreased during 2008 and 2015, suggesting a gradual decrease in use. The ratio of ILD to all reports decreased in those periods. The decrease could be interpreted as the comparatively better safety management of ILD.

\subsubsection{Other Antineoplastic Drugs}

The ILD-reporting patterns were constant for other antineoplastic drugs such as methotrexate, docetaxel, and gemcitabine. These drugs contribute to the accumulation of ILD reports given their longer availability for post-marketing use.

Regarding gemcitabine, the ILD report totals hovered around 90 reports per year after the re-examination period expired. While the ILD reports with the indication of lung cancer were constant during the observation period, the small increase in ILD reporting between 2010 and 2012 was due to the increase in the reports with the indication of pancreatic adenocarcinoma and stage 4 pancreatic cancer. In addition, the small increase between 2014 and 2016 was due to the increase in the reports whose indications were pancreatic cancer with distant metastasis and pancreatic cancer with concomitant use of paclitaxel formulated as albumin-bound nanoparticles. Given that the use of a drug changes with scientific progress in the treatment regimen, the regulators have to be aware of any increase even after the re-examination period has expired.

\subsection{Consideration on Solicited Reports and Spontaneous Reports}

There are interrelating effects between solicited reports and spontaneous reports in pharmacovigilance. We believe that pro-active regulatory requirements, such as the use-results survey during the re-examination period, in addition to the spontaneous reporting system, allow for immediate implementation of regulatory actions with potential clinical benefit. 
For example, the high reporting of ILD may be responsible for the early detection of the importance of the dosing sequence of EGFR-TKIs and nivolumab, which subsequently led to a series of Ministry of Health, Labour and Welfare notifications $[17,18]$. The trigger was the accumulation of the solicited reports and the spontaneous reports of the EGFR-TKIs. In January 2018, the Ministry of Health, Labour and Welfare issued a relevant notification about an interim report of the all-case survey of osimertinib, where 33 ILD cases with a history of nivolumab were identified [19]. In February 2019, the label of osimertinib was updated based on the consideration that the final report of the all-case survey identified that the history of ILD and prior treatment of nivolumab were the risk factors for ILD in the treatment with osimertinib [20]. Given the higher incidence of pre-existing ILD in NSCLC, we must avoid misinterpretation that previously taking NSCLC drugs is a risk factor. The label update was based on the comparison with other covariates, such as previous chemotherapy treatment, World Health Organization performance status, and pulmonary radiation, identifying the risk with specificity [14]. In addition, the effect of previous nivolumab treatment was time dependent, suggesting the biological mechanism.

Meanwhile, the EGFR-TKIs and nivolumab programmed death-ligand 1 (PD-L1) blockade combination underwent scientific scrutiny. In an analogous situation, the durvalumab (another anti-PD-L1 antibody) plus osimertinib arm of the TATTON trial, ILD was markedly high [21]. There is growing concern that treatment with the PD-L1 blockade affects the incidence of EGFR-TKI-induced ILD in patients with NSCLC [22, 23], In particular, given that several EGFR-TKIs and PD-L1 pathway inhibitors are currently on the market, it is important to further elucidate the safest combination or sequence for the use of these therapies.

\section{Limitations}

While this study identified that the regulatory requirement may contribute to high-reporting ILD, other factors could also add to reporting ILD. One prominent example is the higher prevalence and availability of computed tomography (CT) for ILD diagnosis on the back of universal health insurance coverage. Indeed, most of the labels for oncology drugs that contain ILD in the "clinically significant adverse reactions" sections include recommendations for chest $\mathrm{x}$-rays or chest CT scans. The warning section of Afinitol ${ }^{\circledR}$ recommends a CT scan even before starting administration of the drug as well as regularly during treatment. These situations suggest that the use of $\mathrm{CT}$ is routine within clinical practice in Japan.

Another important potential explanation is an underlying pharmacoethnic vulnerability to ILD in Japanese individuals. Epidemiologic data have indicated that the acute exacerbation observed in idiopathic pulmonary fibrosis and drug-induced exacerbation of ILD may be more common in Japan than in the rest of the world [24]. Furthermore, a number of genetic markers have been identified for different disease-associated and drug-induced types of ILD and their prevalence in Japanese patients has been explored. [25-28]. A MUC5B promoter variant was recently reported to be a risk factor of rheumatoid arthritis-associated ILD in a multi-ethnic case series study, but it was under-represented and the relationship was not observed in Asian populations, Japan and China. A prospective study to analyze the relationship between human leukocyte antigen alleles and ILD in Japanese patients with advanced pancreatic cancer revealed that the combination of HLA-B*15:01 and DRB*15:01 was associated with gemcitabine- plus erlotinib-induced ILD. A recent whole genome sequencing study in Japan identified two single nucleotide variants, which showed an association with drug-induced ILD, and other candidate single nucleotide variants for ILD induced by EGFR-TKIs. Future largescale studies are awaited to convincingly determine ethnic differences in the genetics of ILD.

Interstitial lung disease was identified as one of the most outstanding features of Japanese ADR reporting using a disproportionality analysis. Given that the solicited schemes affect disproportionality metrics, we must interpret the data with caution. Accordingly, regulatory agencies try to consolidate the evidence with several schemes. To exclude the false-positives, the causality assessment of the reports is essential. The importance of the causality assessment is exemplified by the fact that a label is revised to contain the risk information when several cases are reported in which the causality between the suspect drug and the adverse effect could be reasonably possible. Another scheme is the post-marketing database investigation, which is used to assess the frequency of ADR occurrence, as a comparison with other drugs, and to differentiate between ADRs and the symptoms of the underlying disease. It also excludes the problem of underreporting. We believe the case reports and the disproportionality analysis are hypothesis generating in the early phase of pharmacovigilance and they should be combined with other evidence as discussed in Sect. 4.2. We have to classify the weight of evidence depending on the situation. The regulatory requirement is intended as a measure to minimize the risk of ILD in Japan; however, the elucidation of the precise mechanism or etiology could provide insights into further safety measures. 


\section{Conclusions}

This study revealed the high rates of ILD reporting derived from mainly the ATC group L drugs, and the solicited report from the use-results survey for those drugs is a contributory factor for high rates of ILD reporting in Japan. We have identified the extent of contribution to reporting from the regulatory scheme. The high reporting of ILD has a clinical benefit, since the solicited reports are of high quality, while accumulating additional evidence for safety. The distinct timeline of the reporting for MAH leads to early awareness and agile pharmacovigilance. This system should be leveraged to respond immediately to the changing landscape of drug use to protect patient safety.

Acknowledgements We thank Dr. Daisaku Sato and Dr. Emiko Kondo for their constructive comments and Saori Soejima for her excellent assistance.

\section{Compliance with Ethical Standards}

Funding No specific grant from any funding agency in the public, commercial, or not-for profit sectors was received for the conduct of this study or the preparation of this article.

Conflict of Interest Eiko Iwasa, Yu Fujiyoshi, Yuki Kubota, Ryota Kimura, Rebecca E. Chandler, Henric Taavola, G. Niklas Norén, and Rika Wakao have no conflicts of interest that are directly relevant to the content of this study.

Data Sharing The Japanese datasets analyzed during the current study are available in the JADER repository (https://www.pmda.go.jp/safety/ info-services/drugs/adr-info/suspected-adr/0005.html).

Open Access This article is licensed under a Creative Commons Attribution-NonCommercial 4.0 International License, which permits any non-commercial use, sharing, adaptation, distribution and reproduction in any medium or format, as long as you give appropriate credit to the original author(s) and the source, provide a link to the Creative Commons licence, and indicate if changes were made. The images or other third party material in this article are included in the article's Creative Commons licence, unless indicated otherwise in a credit line to the material. If material is not included in the article's Creative Commons licence and your intended use is not permitted by statutory regulation or exceeds the permitted use, you will need to obtain permission directly from the copyright holder. To view a copy of this licence, visit http://creativecommons.org/licenses/by-nc/4.0/.

\section{References}

1. Pharmaceutical Affairs Law [in Japanese]. Law number: Act No. 145 of 1960, last version: Amendment of Act No. 108 of 2016. https://elaws.e-gov.go.jp/search/elawsSearch/elaws_search/lsg05 00/detail?lawId=335AC0000000145. Accessed 6 Apr 2020.

2. Good Vigilance Practice ministerial ordinance [in Japanese]. Law number: Act No. 135 of 2004, last version: Amendment of Act No. 124 of 2017. https://elaws.e-gov.go.jp/search/elawsSearch/
elaws_search/lsg0500/detail?lawId=416M60000100135\&opene rCode $=1$. Accessed 6 Apr 2020

3. Wakao R, Taavola H, Sandberg L, Iwasa E, Soejima S, Chandler $\mathrm{R}$, et al. Data-driven identification of adverse event reporting patterns for Japan in VigiBase, the WHO Global Database of Individual Case Safety Reports. Drug Saf. 2019;42(12):1487-98. https ://doi.org/10.1007/s40264-019-00861-y.

4. Koo LC, Clark JA, Quesenberry CP, Higenbottam T, Nyberg F, Wolf MK, et al. National differences in reporting 'pneumonia' and 'pneumonia interstitial': an analysis of the WHO International Drug Monitoring Database on 15 drugs in nine countries for seven pulmonary conditions. Pharmacoepidemiol Drug Saf. 2005;14(11):775-87. https://doi.org/10.1002/pds.1071.

5. Pinheiro L, Blake K, Januskiene J, Yue QY, Arlett P. Geographical variation in reporting interstitial lung disease as an adverse drug reaction: findings from an European Medicines Agency analysis of reports in EudraVigilance. Pharmacoepidemiol Drug Saf. 2016;25(6):705-12. https://doi.org/10.1002/pds.3998.

6. Azuma A, Kudoh S. High prevalence of drug-induced pneumonia in Japan. Jpn Med Assoc J. 2007;50(5):405-11.

7. Kudoh S, Kato H, Nishiwaki Y, Fukuoka M, Nakata K, Ichinose $\mathrm{Y}$, et al. Interstitial lung disease in Japanese patients with lung cancer: a cohort and nested case-control study. Am J Respir Crit Care Med. 2008;177(12):1348-57. https://doi.org/10.1164/ rccm.200710-15010C.

8. Cohen MH, Williams GA, Sridhara R, Chen G, McGuinn WD Jr, Morse D, et al. United States Food and Drug Administration Drug Approval summary: gefitinib (ZD1839; Iressa) tablets. Clin Cancer Res. 2004;10(4):1212-8.

9. Skeoch S, Weatherley N, Swift AJ, Oldroyd A, Johns C, Hayton C, et al. Drug-induced interstitial lung disease: a systematic review. J Clin Med. 2018;7(10):356. https://doi.org/10.3390/ jem7100356.

10. Ishiguro C, Misu T, Iwasa E, Izawa T. Analysis of safety-related regulatory actions by Japan's pharmaceutical regulatory agency. Pharmacoepidemiol Drug Saf. 2017;26(11):1314-20. https://doi. org/10.1002/pds.4252.

11. Lagerlund O, Strese S, Fladvad M, Lindquist M. WHODrug: a global, validated and updated dictionary for medicinal information. Ther Innov Regul Sci. 2020. https://doi.org/10.1007/s4344 1-020-00130-6.

12. Juhlin K, Star K, Noren GN. A method for data-driven exploration to pinpoint key features in medical data and facilitate expert review. Pharmacoepidemiol Drug Saf. 2017;26(10):1256-65. https://doi.org/10.1002/pds.4285.

13. Shah RR. Tyrosine kinase inhibitor-induced interstitial lung disease: clinical features, diagnostic challenges, and therapeutic dilemmas. Drug Saf. 2016;39(11):1073-91. https://doi. org/10.1007/s40264-016-0450-9.

14. AstraZeneca KK. Final report of use-results survey of tagrisso. 2019. https://www.pmda.go.jp/files/000228398.pdf. Accessed 6 June 2020

15. Nakagawa K, Kudoh S, Ohe Y, Johkoh T, Ando M, Yamazaki N, et al. Postmarketing surveillance study of erlotinib in Japanese patients with non-small-cell lung cancer (NSCLC): an interim analysis of 3488 patients (POLARSTAR). J Thorac Oncol. 2012;7(8):1296-303. https://doi.org/10.1097/JTO.0b013e3182 $598 \mathrm{abb}$.

16. Saijo N, Kato H, Kudo S, Tada H, Tamura T, Hayakawa K, et al. Guideline for using gefitinib [in Japanese]. 2005. https://www. pmda.go.jp/files/000145266.pdf. Accessed 6 June 2020.

17. MHLW. Precautions relating to interstitial lung disease during administration of epidermal growth factor receptor tyrosine kinase inhibitors [in Japanese]. MHLW, Tokyo. 2016. https://www.pmda. go.jp/files/000213244.pdf. Accessed 6 June 2020. 
18. MHLW. Pharmaceuticals and medical devices safety information no. 336. September 2016. https://www.pmda.go.jp/files/00021 4024.pdf. Accessed 6 June 2020.

19. MHLW. Notification about interim report of the all-case survey of osimertinib [in Japanese]. MHLW, Tokyo. 2018. https://www. mhlw.go.jp/file/06-Seisakujouhou-11120000-Iyakushokuhinky oku/0000192974.pdf. Accessed 6 June 2020.

20. MHLW. Notification about osimertinib use-results survey [in Japanese]. MHLW, Tokyo. 2019. https://www.mhlw.go.jp/conte nt/11120000/000484135.pdf. Accessed 6 June 2020.

21. Oxnard GR, Yang JC, Yu H, Kim SW, Saka H, Horn L, et al. TATTON: a multi-arm, phase Ib trial of osimertinib combined with selumetinib, savolitinib, or durvalumab in EGFR-mutant lung cancer. Ann Oncol. 2020;31(4):507-16. https://doi.org/10.1016/j. annonc.2020.01.013.

22. Oshima Y, Tanimoto T, Yuji K, Tojo A. EGFR-TKI-associated interstitial pneumonitis in nivolumab-treated patients with nonsmall cell lung cancer. JAMA Oncol. 2018;4(8):1112-5. https:// doi.org/10.1001/jamaoncol.2017.4526.

23. Schoenfeld AJ, Arbour KC, Rizvi H, Iqbal AN, Gadgeel SM, Girshman J, et al. Severe immune-related adverse events are common with sequential PD-(L)1 blockade and osimertinib. Ann Oncol. 2019;30(5):839-44. https://doi.org/10.1093/annonc/mdz077.

24. Saito S, Lasky JA, Hagiwara K, Kondoh Y. Ethnic differences in idiopathic pulmonary fibrosis: the Japanese perspective. Respir
Investig. 2018;56(5):375-83. https://doi.org/10.1016/j.resin v.2018.06.002.

25. Namba N, Kawasaki A, Sada KE, Hirano F, Kobayashi S, Yamada $\mathrm{H}$, et al. Association of MUC5B promoter polymorphism with interstitial lung disease in myeloperoxidase-antineutrophil cytoplasmic antibody-associated vasculitis. Ann Rheum Dis. 2019;78(8):1144-6. https://doi.org/10.1136/annrheumdis-2018214263.

26. Juge PA, Lee JS, Ebstein E, Furukawa H, Dobrinskikh E, Gazal $\mathrm{S}$, et al. MUC5B promoter variant and rheumatoid arthritis with interstitial lung disease. N Engl J Med. 2018;379(23):2209-19. https://doi.org/10.1056/NEJMoa1801562.

27. Nishimura M, Toyoda M, Takenaka K, Imamura Y, Chayahara N, Kiyota N, et al. The combination of HLA-B*15:01 and DRB1*15:01 is associated with gemcitabine plus erlotinibinduced interstitial lung disease in patients with advanced pancreatic cancer. Cancer Chemother Pharmacol. 2016;77(6):1165-70. https://doi.org/10.1007/s00280-016-3026-6.

28. Udagawa C, Horinouchi H, Shiraishi K, Kohno T, Okusaka T, Ueno $\mathrm{H}$, et al. Whole genome sequencing to identify predictive markers for the risk of drug-induced interstitial lung disease. PLoS ONE. 2019;14(10):e0223371. https://doi.org/10.1371/journ al.pone. 0223371 . 